\title{
PROYECTO EDNA. \\ LOGROS Y DESAFÍOS DE UNA AGENDA PERMANENTE
}

\author{
EDNA PROJECT. \\ ACHIEVEMENTS, CHALLENGES OF A PERMANENT AGENDA
}

\author{
Marisol Gutiérrez Rojas ${ }^{1}$ \\ marigu62@hotmail.com
}

Fecha de recepción: 12 mayo 2015 - Fecha de aceptación: 15 diciembre 2015

\begin{abstract}
Resumen
El presente artículo sistematiza la experiencia del proyecto Estado de los derechos de la niñez y la adolescencia (EDNA): sus orígenes, modelo de gestión, alianzas, aportes y desafíos en una agenda comprometida con la protección y promoción de los derechos de esta población. El documento hace hincapié en el periodo 2007-2012; en la elaboración y contenidos de siete informes que evidencian el devenir histórico y las inflexiones en materia de derechos de la población menor de edad; y en el papel de la academia para analizar, liderar, conjuntar y propiciar esfuerzos intersectoriales, interinstitucionales e interdisciplinarios en beneficio de este grupo poblacional.

Palabras clave: niñez-adolescencia-derechos-investigación-acción social.
\end{abstract}

\begin{abstract}
This article systematizes the experience of the project "State of the rights of children and adolescence" (EDNA): its origins, management model, alliances, contributions and challenges of an agenda committed to protect and promote the rights of this population. The document emphasizes during the period 20072012; the preparation and contents of seven reports showing the historical development and inflections on the rights of child population; and the role of the academy to analyze, lead, bring together and propitiate joint efforts within intersectoral, interdisciplinary and inter-agencies on behalf of this group. Key words: childhood, adolescence, investigation, rights, social action.
\end{abstract}

\section{Introducción}

En el 2000, la Universidad de Costa Rica (UCR), consecuente con sus principios de universidad pública y humanista, articula un interesante diálogo con el Fondo de las Naciones Unidas para la Infancia (Unicef), con miras a una mayor y vigorosa atención a la población menor de edad del país.

Dos hitos altamente significativos: la ratificación de la Convención sobre los Derechos del Niño (1989) y la creación y aprobación del Código de la Niñez y la Adolescencia (1998) -los que inciden en

1 Docente de la Escuela de Estudios Generales en la Sección de Comunicación y Lenguaje y de Seminarios de Realidad Nacional. 
un cambio de paradigmas, de la situación irregular a la protección integral de la niñez y la adolescencia- ${ }^{2}$ favorecen la conjunción de las preocupaciones universitarias y de Unicef. Estas pronto se concretan en la firma de un convenio (2002) ${ }^{3}$ que impulsa la creación del Programa Interdisciplinario de Estudios y Acción Social de los Derechos de la Niñez y la Adolescencia (Pridena), ${ }^{4}$ entre cuyos objetivos específicos destaca la elaboración de un Estado de los derechos de la niñez y la adolescencia. La publicación de este primer informe ${ }^{5}$ marcará un hito, pues un documento de esta naturaleza no existía en América Latina.

Documentar una experiencia académica es un deber universitario, Máxime si, como en el caso de la que ocupa las siguientes páginas, se involucran los tres pilares de la Universidad: acción social, investigación y docencia, hay esfuerzos interdisciplinarios, una labor sostenida que supera una década, y se atiende un tema donde la Universidad asume un compromiso político a nivel nacional en la defensa y promoción de los derechos humanos de la población menor de edad.

Con ese cometido, en las páginas siguientes se hará un recorrido por el devenir e impacto del proyecto EDNA, con un particular énfasis en las tareas acometidas del 2007 al 2012, cuando la UCR y Unicef concertaron un viraje estratégico en la concepción y desempeño de este proyecto. El objetivo central para el que se concibió el proyecto, elaborar un estado de la situación de los derechos de las personas menores de edad, seguirá siendo relevante, pero no el único. El informe pasará a ser una publicación bienal, en el marco de un proyecto caracterizado como una amplia estrategia de promoción y defensa de los derechos de la niñez y la adolescencia, para un horizonte de cinco años: 2007-2012.

\section{¿Qué es el proyecto EDNA?}

A lo interno de la UCR, el Estado de los Derechos de la Niñez y la Adolescencia (EDNA) es un proyecto de Extensión Docente, ${ }^{6}$ enmarcado dentro del Pridena. El proyecto, de carácter interdisciplinario, en la actualidad está inscrito en la Escuela de Estudios Generales (EEG) y cuenta con la colaboración de la Escuela de Trabajo Social (ETS), así como de una importante red de investigadoras e investigadores de esta Universidad y de otras instancias de la sociedad costarricense.

El objetivo central del proyecto EDNA es la coordinación académica de la elaboración, divulgación y seguimiento estratégico de un informe sobre el curso del cumplimiento de los derechos de la niñez y la adolescencia en Costa Rica, en consonancia con lo que establece el Código de la Niñez y la

2 El paradigma de protección integral de niños, niñas y adolescentes supone realizar en los países que han ratificado la Convención, transformaciones de diverso tipo (jurídicas, institucionales y culturales) con el fin de garantizar el cumplimiento cabal de los derechos. Aun cuando al Estado le corresponde velar y crear las condiciones para el disfrute pleno de los derechos humanos y la aplicación de la legislación en la materia, esta responsabilidad no es exclusiva. También son responsables las personas adultas individualmente, el sector privado, las organizaciones no gubernamentales y las organizaciones sociales y comunitarias nacionales o locales (UCR y Unicef, 2003).

3 En el 2002 se firma un Convenio que rige hasta el año 2010; este luego es renovado hasta el 2013, y a su término, se firma un memorándum de entendimiento que rige del 2013 hasta el 2018.

4 El Pridena se crea en el 2002, como un Proyecto de Extensión Docente (ED-913) de la Escuela de Trabajo Social (ETS) de la Universidad de Costa Rica, inscrito en la Vicerrectoría de Acción Social. Su objetivo es: "Constituirse en un espacio de producción, transferencia y divulgación de conocimiento teórico-práctico en materia de niñez y adolescencia, que le permita convertirse en una plataforma de asistencia técnica y de apoyo a las reformas jurídicas, institucionales y sociales suscitadas en el ámbito nacional y regional" (Pridena, 2008, p.6).

5 En la creación del informe EDNA participó también, de forma destacada, la Facultad Latinoamericana de Ciencias Sociales (Flacso). La generación del primer informe (2001), es anterior a la firma del convenio, y en razón de su importancia y del tratamiento del tema de los derechos de la niñez y la adolescencia en el marco de una alianza con la academia, es que se formaliza la relación UCR/Unicef y se abre así un amplio horizonte de acciones conjuntas que se prolongan hasta la actualidad.

6 Para efectos de clasificación interna, el proyecto es el ED-389. 
Adolescencia (CNA) y otros cuerpos normativos vinculados. Este Informe se viene desarrollando desde el 2001, con el respaldo financiero, logístico y conceptual de la Vicerrectoría de Acción Social (VAS) y Unicef (con apoyo ocasional de la Vicerrectoría de Investigación (VI).

El Informe EDNA se realiza de forma conjunta con el proyecto Sistema de Información Estadística en Derechos de Niñez y Adolescencia (Siedna), de cuyo esfuerzo compartido surge un instrumento -cualitativo y cuantitativo- valioso y pertinente para visualizar los problemas, vacíos, avances o retrocesos del país con respecto a la situación de niños, niñas y adolescentes; así como temáticas particulares,que a veces no están en agenda, a pesar de su relevancia.

El Informe EDNA ha buscado incidir de manera prioritaria en el área político-gubernamental, los mandos medios y los funcionarios de campo de la institucionalidad pública que integra el Sistema Nacional de Protección Integral (SNPI), la sociedad civil y la comunidad universitaria. El documento es un insumo para quienes formulan política pública y toman decisiones a este nivel, a la vez que constituye un material de consulta para investigadores(as), docentes, estudiantes y profesionales de diversos campos quienes, de manera directa o indirecta, atienden lo concerniente a derechos de la población menor de edad.

De manera particular, en diversos momentos del proceso de elaboración y divulgación del informe EDNA, se ha trabajado de forma activa con niñas, niños y adolescentes (en especial los de las juntas de protección de la niñez y la adolescencia),en consonancia con su condición de sujetos de derechos, de manera que se evidencie cuáles temas de protección de sus derechos priorizan y cómo valoran los eventuales avances, vacíos y desafíos en este tema.

En el 2007, en el marco de una interesante reflexión sobre la incidencia del informe y del compromiso asumido por la UCR y Unicef en la promoción y defensa de los derechos de las personas menores de edad, el proyecto EDNA se modifica para hacer efectiva una amplia estrategia de trabajo en redes, que fortalece las acciones interinstitucionales, intersectoriales e interdisciplinarias, para un horizonte de cinco años: 2007-2012, donde el informe, como documento, será un elemento central, de publicación bienal, al que se sumarán acciones puntuales de investigación, acción social y docencia.

\section{Informe EDNA}

El Informe Estado de los derechos de la niñez y la adolescencia representa un esfuerzo sostenido de la UCR -con apoyo de Unicef- a lo largo de casi tres quinquenios, que ha superado los cambios de administración (nacionales o institucionales), las variaciones en las políticas para este sector poblacional y la demanda de recursos humanos y materiales que una acción como esta implica, para dar continuidad a un informe que, a la fecha de este artículo, suma siete (7) ediciones. Esto, sin duda, evidencia su compromiso como garante del bien común y del mejoramiento de la calidad de vida de las personas. ${ }^{7}$

\section{Recuento histórico}

Invariablemente con el apoyo de la VAS y del Pridena (en consecuencia de la ETS), el proyecto EDNA ha tenido un edificante devenir en la vida de la UCR. Dado su carácter interdisciplinario, ha sido objeto de coordinación de distintas unidades académicas. ${ }^{8}$ Esta circunstancia ha sido una excelente oportunidad para sumar esfuerzos en ámbitos propios de cada unidad, valga decir: políticas públicas,

7 Castillo, Arias y Zúñiga (2011) hacen un recuento de este compromiso de la UCR al referir acciones como la profesionalización de docentes en carreras que atienden esta población, la atención en los centros y casas infantiles así como en la escuela y el Liceo Laboratorio; la creación del programa institucional Pridena y el apoyo a la cartera de proyectos que este cobija -entre estos el EDNA-; y su participación en la Red Interinstitucional para la niñez y la adolescencia (RINA) y en la coalición Universidades para la Infancia y la Adolescencia.

8 La unidad ejecutora en general ha sido la misma a la que pertenece quien asume la coordinación del proyecto y se brinda la carga académica para tales efectos. 
justicia social e institucionalidad democrática, inclusión y equidad de género, temas sociales, colectivos y basados en diferencias etarias, entre otros. Asimismo, estos diálogos han favorecido la conformación de una estructura organizativa interdisciplinaria, que enriquece la visión del tema de estudio, y la vez, la estructuración de un equipo básico estable de académicos y académicas que asuma la conducción general del proyecto, lo fortalezca y le dé sostenibilidad.

En la actualidad, la unidad ejecutora del proyecto EDNA es la Escuela de Estudios Generales. A continuación, se presenta un cuadro que relata este devenir.

Unidades académicas donde ha estado inscrito el proyecto EDNA

\begin{tabular}{lcll}
\hline \multicolumn{1}{c}{ Unidad académica } & Años & \multicolumn{1}{c}{ Coordinador(a) } & \multicolumn{1}{c}{ Título } \\
\hline Escuela de Ciencias Políticas & 1999 & Erick Hess Araya & $\begin{array}{l}\text { Estado nacional de los derechos de } \\
\text { la niñez y la adolescencia }\end{array}$ \\
\hline $\begin{array}{l}\text { Maestría Regional en Estudios } \\
\text { de la Mujer }\end{array}$ & 2000 & Monserrat Sagot Rodríguez & $\begin{array}{l}\text { Estado nacional de los derechos de } \\
\text { la niñez y la adolescencia }\end{array}$ \\
\hline Escuela de Trabajo Social & 2002 & Sonia Solís Umaña & $\begin{array}{l}\text { Estado de los derechos de la niñez y } \\
\text { la adolescencia }\end{array}$ \\
\hline Escuela de Nutrición & 2005 & $\begin{array}{l}\text { Leda Muñoz García (con la } \\
\text { colaboración de } \\
\text { Juan Diego Trejos, Monserrat } \\
\text { Sagot y Doris Sossa) }\end{array}$ & $\begin{array}{l}\text { Estado de los derechos de la niñez y } \\
\text { la adolescencia }\end{array}$ \\
\hline Escuela de Nutrición & & $\begin{array}{l}\text { Marisol Gutiérrez Rojas y } \\
\text { Nancy Piedra Guillén }\end{array}$ & $\begin{array}{l}\text { Estado de los derechos de la niñez y } \\
\text { la adolescencia }\end{array}$ \\
\hline Escuela de Trabajo Social & 2006 & Marisol Gutiérrez Rojas & $\begin{array}{l}\text { Estado de los derechos de la niñez y } \\
\text { la adolescencia }\end{array}$ \\
\hline Escuela de Estudios Generales & $2007-2008-2013$ & $\begin{array}{l}\text { Marisol Gutiérrez Rojas } \\
\text { Estado de los derechos de la niñez y } \\
\text { la adolescencia }\end{array}$ \\
\hline
\end{tabular}

Fuente: Elaboración propia con base en documentos de archivos de la Vicerrectoría de Acción Social.

\section{Recuento temático}

El informe EDNA ha permitido visualizar, a través de sus ediciones, los problemas, avances o retrocesos relativos a aspectos generales del país y temáticas particulares con respecto a la situación de los derechos de niños, niñas y adolescentes; y revelar importantes vacíos de investigación que convendría atender en la academia, por ejemplo, desde proyectos de graduación.

Algunos temas se han abordado de manera recurrente, valga decir: la situación demográfica, el Sistema Nacional de Protección Integral, la reforma del Patronato Nacional de la Infancia(PANI), la familia, la participación social y los gobiernos locales);sin embargo, no ha habido un hilo conductor o de continuidad de un informe a otro, siendo algunas ediciones unidades en sí mismas. No obstante, cabe subrayar que el VI EDNA constituyó una experiencia particular al ser un análisis a diez años del Código de la Niñez y la Adolescencia, así como el VII EDNA que, por su parte, se vertebró a la luz de los seis ejes de la Política Nacional de Niñez y Adolescencia (PNNA) 2009- 2021. Temas estratégicos fueron acotados a la vez por el enfoque de derechos ${ }^{9}$ y los Objetivos de Desarrollo del Milenio, constituyéndose

$9 \quad$ El enfoque de derechos humanos es una perspectiva de análisis y de acción social que identifica a la persona en su dimensión colectiva e individual en la sociedad y concibe los derechos como los medios para garantizar el acceso, las capacidades y 
en el marco de un análisis interdisciplinario que enfatizó en el cumplimiento de compromisos y en el seguimiento de las acciones de los distintos actores sociales y políticos de nuestro país, sobre todo los gobiernos locales.

Mención especial merece el anexo estadístico de los distintos informes, pues constituye un instrumento de alta utilidad para entender los capítulos y también, para señalar vacíos en indicadores, como en el caso del VI Informe, donde queda de manifiesto la ausencia de cifras vinculadas al cumplimiento de algunos derechos, tal es el caso del derecho a la cultura.

La información registrada en los cuadros que se presentan a continuación ilustra lo referido.

$$
\text { Énfasis de los temas tratados en los Informes EDNA (I al VII) }
$$

\begin{tabular}{|c|c|c|}
\hline AÑO & Tema principal & Ejes de análisis \\
\hline I & $\begin{array}{l}\text { El ciclo de vida, la valoración de la autonomía } \\
\text { de los grupos sociales y la eficacia o desempeño } \\
\text { institucional. }\end{array}$ & $\begin{array}{l}\text { Situación, cumplimiento de los derechos, protección local, } \\
\text { desempeño institucional. }\end{array}$ \\
\hline II & $\begin{array}{l}\text { Logros, opciones y dificultades que experimen- } \\
\text { tan niñas, niños y adolescentes en el ejercicio y } \\
\text { ampliación de sus derechos, calidad de vida y } \\
\text { aporte social. }\end{array}$ & $\begin{array}{l}\text { Grupos de edad y problemas específicos. } \\
\text { Situación de la población de } 0 \text { a } 5 \text { años. } \\
\text { Relación entre el trabajo infanto -juvenil y el derecho } \\
\text { a la educación. } \\
\text { Condiciones de explotación sexual comercial. }\end{array}$ \\
\hline III & $\begin{array}{l}\text { Inversión social: instrumento para el cumplimien- } \\
\text { to de los derechos. }\end{array}$ & $\begin{array}{l}\text { Asignaciones presupuestarias, gerencia y efectividad. } \\
\text { Acceso a programas sociales, estimación de la inversión } \\
\text { social, pobreza y equidad, servicios sociales y gobiernos } \\
\text { locales. }\end{array}$ \\
\hline IV & $\begin{array}{l}\text { Avances en implementación de CNNA y el reco- } \\
\text { nocimiento de los derechos. }\end{array}$ & $\begin{array}{l}\text { Sistema Nacional de Protección Integral. } \\
\text { Asimetrías en la inversión social según edad, región zona, } \\
\text { sexo, ingreso. } \\
\text { Tendencias y características de las familias. }\end{array}$ \\
\hline $\mathrm{V}$ & $\begin{array}{l}\text { Seguimiento a la situación de los derechos de } \\
\text { niñas, niños y adolescentes. }\end{array}$ & $\begin{array}{l}\text { Ciudadanía, educación general, educación secundaria } \\
\text { Miradas de las y los adolescentes. } \\
\text { Afectividad. } \\
\text { Reforma del PANI, Sistema Nacional de Protección } \\
\text { Integral. }\end{array}$ \\
\hline VI & $\begin{array}{l}\text { Desempeño del país a diez años de la puesta en } \\
\text { vigencia del CNA. }\end{array}$ & $\begin{array}{l}\text { Del paradigma asistencial al paradigma de protección } \\
\text { integral. }\end{array}$ \\
\hline VII & $\begin{array}{l}\text { Política Nacional de Niñez y Adolescencia } \\
\text { 2009- } 2021 .\end{array}$ & Ejes y lineamientos estratégicos de la PNNA. \\
\hline
\end{tabular}

Fuente: Elaboración propia con base en los informes EDNA.

ejercicio de la igualdad, bienestar y libertad (Guendel, Barahona y Bustelo, 2005). En relación con la niñez y la adolescencia, el primer Informe EDNA genera un debate teórico y conceptual en torno a este enfoque, reconociendo a esta población como sujetos activos que están en capacidad de establecer relaciones reflexivas -acordes con su ciclo de vida- con las personas adultas; pero que también son personas diferentes, que experimentan necesidades singulares marcadas por las determinaciones propias de las etapas de formación temprana que están viviendo. Es decir, el enfoque procura retomar las particularidades del grupo poblacional y propone el desarrollo de una visión integral de los problemas sociales. 
Temáticas recurrentes de cada Informe EDNA

\begin{tabular}{ll}
\hline \multicolumn{1}{c}{ Principales temas de los EDNA } & Tratados en los EDNA indicados \\
\hline Situación general de los derechos de la niñez y la adolescencia & I, II, IV,V \\
\hline Situación demográfica & I, III, IV, V \\
\hline El Sistema Nacional de Protección Integral & I, III, IV, V,VI,VII \\
\hline La reforma PANI & I, III, IV, V \\
\hline Familia & I, II, III \\
\hline Gobiernos locales & III, IV, VI,VII \\
\hline Ciudadanía y participación & I, V, VI \\
\hline Inversión social & III, IV \\
\hline Programas sociales & III, IV \\
\hline Explotación sexual & II \\
\hline Trabajo infantil & II \\
\hline Pobreza y equidad & III \\
\hline Educación desde la mirada de las personas adolescentes & V, VI \\
\hline Educación secundaria & V,VI \\
\hline
\end{tabular}

Fuente: Elaboración propia con base en los informes EDNA.

La información señalada en los cuadros arriba expuestos también permite leer los vacíos de investigación en asuntos altamente sensibles, como es el caso del derecho a la educación sexual integral de las personas menores de edad, la carencia y precariedad de los servicios de salud especializados para adolescentes, situaciones como el abuso sexual infantil, la trata de personas o el embarazo en la adolescencia. Asimismo, debe brindarse atención a poblaciones vulnerables, como los menores de edad en condición de discapacidad, la primera infancia y la niñez en útero; además de profundizar en el tema de interculturalidad, que permita atender las necesidades de los menores de edad indígenas y migrantes, así como otros derechos a veces no considerados "urgentes", como el de la cultura y la recreación.

En esta línea, el informe EDNA busca contribuir en la consolidación de un ejercicio de reflexión téorica, de analisis contextual y situacional de la niñez y la adolescencia, de carácter permanente y esfuerzos conjuntos, tanto desde la academia como desde la institucionalidad y la sociedad civil, por lo que también ha insistido en la necesidad de asumir, de manera vigorosa, un desafío pendiente, como es el fomento de una cultura de evaluación y monitoreo de las acciones ejecutadas, a nivel nacional, destinada a brindar una protección integral a niños, niñas y adolescentes, que permita medir los avances e impacto en la materia.

\section{Estrategia metodológica}

En el 2007 se redefinen las tareas del proyecto en el marco de una estrategia metodológica tendiente a posicionar el informe EDNA (documento), como un producto específico dentro de un amplio proceso de investigación y acción social sobre el tema de derechos de niñez y adolescencia a lo largo de un quinquenio comprendido entre 2007-2012.Sin embargo, no es ajena la preocupación de la UCR por ampliar el estudio a la población joven (comprensible dado que este segmento es la esencia del 
estudiantado universitario), para lo cual habrá de considerarse en el análisis lo establecido en la Ley de la Persona Joven, interés que, como se verá más adelante, será abordado de manera exitosa.

La estrategia metodológica diseñada se define en un ámbito estructurado por el trabajo en redes de apoyo que se articulan alrededor tres área de trabajo interrelacionadas. A saber:

1. Área de reflexión, investigación y diagnóstico. Esta busca la formación de una red de investigadores(as), expertos(as), consultores(as) provenientes de institutos y centros de investigación de la UCR y de otras universidades, instituciones nacionales y otros entes que se interesan en el desarrollo de investigaciones, diagnósticos y reflexiones de calidad académica y científica.

2. Área de participación, prospección y sensibilización.Esta área persigue conformar una red políticoinstitucional que aglutine a personas que, en representación de su institución u organización, puedan coordinar acciones con la UCR, por medio del proyecto EDNA y del Pridena, con el afán de retroalimentar y generar las posibilidades concretas de acción a favor de la niñez y la adolescencia.

3. Área de proyección, información, divulgación y seguimiento. En este marco se busca la formación de una red de comunicación integrada por personas e instancias que apoyen en la difusión del tema de derechos de la niñez y la adolescencia y, particularmente, del proyecto EDNA, así como en la generación de productos que potencien la proyección y posicionamiento de estos esfuerzos.

En la siguiente figura se muestra la articulación de las distintas áreas referidas: 


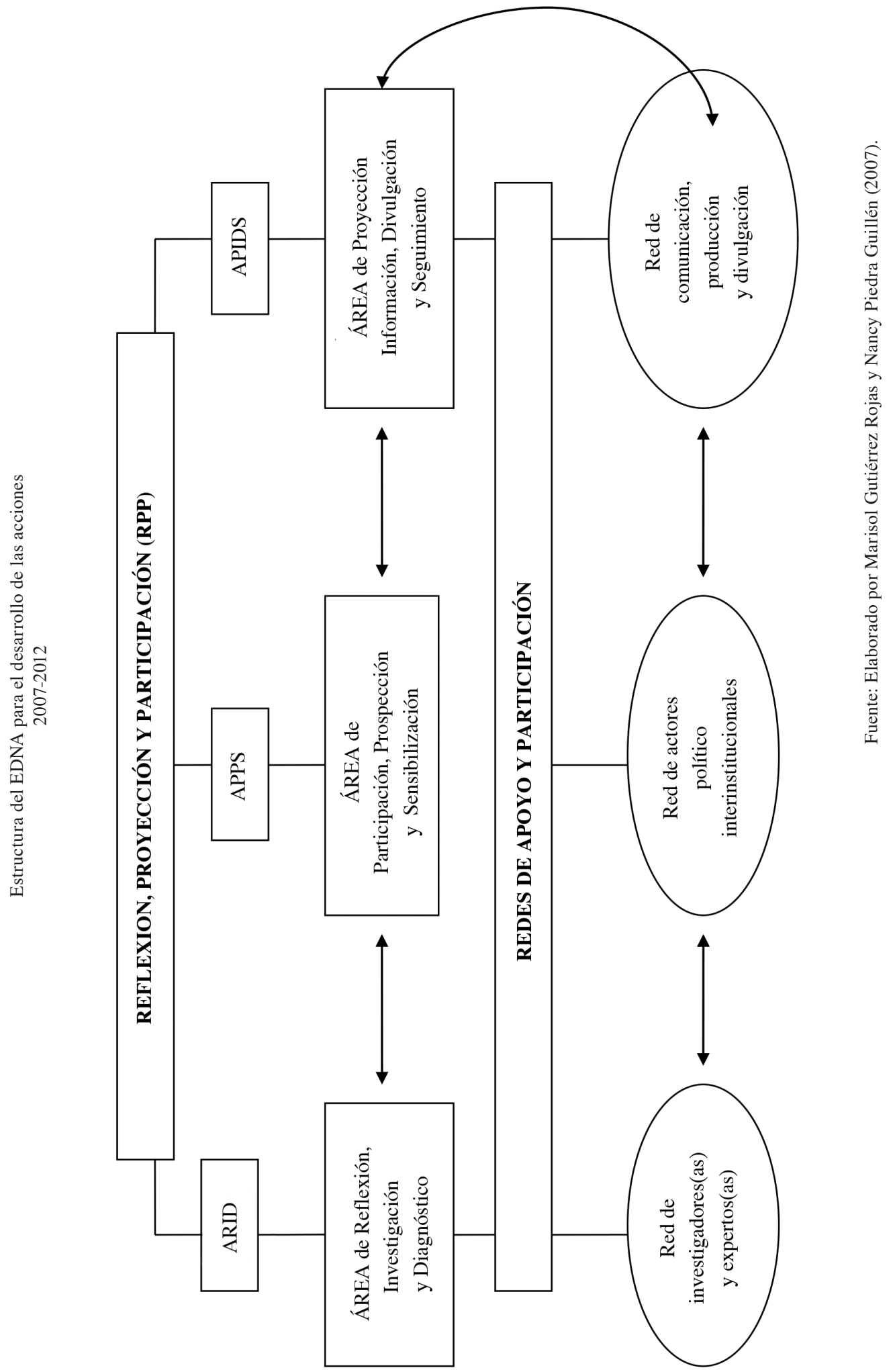


Estas tres áreas, que tienen su especificidad, fungen a la vez como espacios de consulta del proceso de elaboración y seguimiento estratégico del informe EDNA, el que constituye, en sí mismo, una cuarta área de trabajo.

Para efectos de su elaboración, se diseña una estructura de gestión simple pero eficaz,que articula su trabajo en tres momentos: un antes (presupuesto, diseño y tiraje del informe, identificación y contratación del equipo de investigación) $;^{10}$ un durante (investigación, producción del documento en sus distintas versiones y la presentación oficial); un después (seguimiento estratégico del Informe en el que se integran estrategias de divulgación, de sensibilización y capacitación sobre los contenidos). La estructura de gestión está conformada por:

\begin{tabular}{ll}
\hline Coordinación & $\begin{array}{l}\text { Académica (UCR) } \\
\text { Técnica (Unicef) }\end{array}$ \\
\hline \multirow{2}{*}{ Equipo de investigación } & $\begin{array}{l}\text { De consultores(as) a investigadores(as) } \\
\text { Enlaces académicos }\end{array}$ \\
\hline \multirow{3}{*}{ Comité técnico } & Un representante de Unicef, del PANI, de la sociedad civil, designado por elC- \\
& NNA, y un representante de la UCR, en este caso, quien ejerza la Coordinación \\
& Académica \\
Consejo editorial & Un representante de la DHR como observador \\
\hline
\end{tabular}

Fuente: Elaboración propia.

En correspondencia con los objetivos de cada área de trabajo, de forma paralela se emprenden otras acciones puntuales de investigación, análisis y debate -con diversos formatos: mesas redondas, talleres, grupos de discusión, tesinas-sobre temas importantes para los cuales no se tiene suficiente información, y que implica adentrarse en tabúes o en problemáticas que muy recientemente han sido consideradas, tal es el caso del sexting, el bullying, la anorexia y la bulimia, el suicidio en personas adolescentes o el derecho a una educación sexual integral.

En acción social se pone el énfasis en lograr incidir en el medio nacional a través de campañas y acciones concretas, utilizando diversos medios de comunicación, en especial los de la UCR (radio, prensa, televisión, web), con el fin de divulgar e informar acerca de los principales problemas y retos en el tema de la defensa y el ejercicio de los derechos de la niñez y la adolescencia.

Finalmente, en docencia se emprenden esfuerzos, sobre todo con la Escuela de Estudios Generales, para considerar los resultados de los informes en las discusiones de clase, en algunos Repertorios y en los proyectos de investigación que desarrolla el estudiantado.

\section{En el seno de las humanidades}

Tanto el viraje estratégico del proyecto EDNA como su inscripción en el 2009 en la Escuela de Estudios Generales, resultan ser una coyuntura significativa para el fortalecimiento de esta acción académica.

10 El presupuesto asignado a la elaboración del informe EDNA se compone de dos partes: una asignada por Unicef para efectos de la contratación del equipo de investigación, el tiraje de 500 ejemplares físicos de aproximadamente 300-350 páginas, 200 ejemplares en CD (además de las versiones en la web) y la otra asignada por la UCR en cargas académicas para la Coordinación del Informe, el apoyo humano, material y logístico de Pridena, de los enlaces académicos y para el seguimiento estratégico del documento (divulgación, capacitación). Acciones puntuales son apoyadas también por el PANI. 
Recuérdese que ya en el 2007 la Universidad había expresado su preocupación por ampliar el estudio sobre la situación de los derechos de las personas menores de edad a la población joven, en tanto que la Escuela de Estudios Generales incorpora el proyecto argumentando la vocación humanística y el interés por el conocimiento y la vivencia de los derechos humanos que caracteriza esta Escuela; su conformación interdisciplinaria; y su condición de unidad académica que recibe una población estudiantil que, por su condición etaria (parte de ella puede considerarse aún adolescente), constituye un agente de cambio relevante para el trabajo en la promoción y defensa de los derechos humanos, en especial de las personas menores de edad.

En este marco, sobresale una iniciativa innovadora: la Semana joven. Fortalecido ahora el enfoque de derechos con el enfoque de juventudes, se abre un espacio de acciones interdisciplinarias e interinstitucionales donde las y los jóvenes reflexionan sobre sus principales temas de interés y se visibilizan como actores estratégicos del desarrollo nacional. El grado de aceptación de la actividad permite la realización de tres ediciones y la participación activa del Consejo de la Persona Joven (CPJ), ${ }^{11}$ que se suma al histórico vínculo con Unicef. El tema central del informe mundial de este organismo constituye cada año el eje transversal de la actividad. Valga decir que se trabaja en los temas: adolescencia como una época de oportunidades (2011), niñez y adolescencia en el mundo urbano (2012), y niños y niñas con discapacidad (2013).

La Semana Joven, además, se convierte en una acción relevante para la Escuela de Estudios Generales, pues sus estudiantes encuentran una oportunidad para socializar, más allá del aula, aquellos diseños de investigación generados en un espacio propio de su programa de estudios destinado al quehacer investigativo, que tuvieran relación con el ejercicio de sus derechos, tales como: uso y apropiación de espacios públicos por parte de las personas jóvenes, la trata de personas adolescentes para la explotación sexual comercial, la llamada generación ni-ni, la exclusión educativa y laboral, entre otros; así como las investigaciones efectuadas en el Repertorio Reproducción, sexualidad y humanismo, que son plasmadas en atractivos afiches.

La Semana Joven logra impactar no solo a estudiantes universitarios, sino también a colegiales de diversas áreas del país que llegan a las actividades, pues abre un espacio de participación y visibilización inclusiva, donde las voces adolescentes y jóvenes se validan, se genera sentido de pertenencia y se socialicen sus inquietudes y sus producciones académicas; en buenas cuentas, la Semana Joven les ofrece una experiencia de educación en y para los derechos humanos.

\section{Alianzas, impacto y proyección}

La experiencia acumulada a lo largo de esta estrategia quinquenal revela un fortalecimiento en las alianzas y el trabajo en red. Aquí, es significativo el trabajo con el Consejo Nacional de la Niñez y la Adolescencia (CNNA).

El Código de la Niñez y la Adolescencia señala como funciones de la Secretaría Técnica del CNNA, en su artículo 178, inciso c: "Formular un estudio anual sobre el estado de los derechos de la niñez y la adolescencia. Para realizarlo, gestionará la participación de otras instituciones dedicadas al estudio de esta materia, en especial las universidades".

En consonancia con este señalamiento, la UCR, en alianza con Unicef, ha venido aportando insumos a partir de la elaboración del Informe EDNA, tarea que ha sido de alta estima para el CNNA. En el 2007, el Consejo y la UCR concertaron esfuerzos para llevar a cabo la tarea del informe EDNA, asumiendo el Consejo un papel de órgano consultivo. En el 2009, el CNNA acuerda respaldar formalmente la tarea que ha venido haciendo la UCR al reconocer su papel de Coordinadora Académica del

11 En el 2014 se firma un Convenio Marco de Cooperación entre la UCR y el Consejo Nacional de la Política Pública de la Persona Joven. 
Informe, ${ }^{12}$ con lo que ratifica que la presencia de la Universidad garantiza transparencia y probada calidad de investigación.

Como es evidente, este suceso fortalece el cumplimiento de los mandatos del Código desde la academia, pero también, favorece la incidencia en la política pública, al habilitarse un espacio permanente de consulta e interlocución entre ambas instancias.

A esta alianza se suman otras propias de la UCR: unidades académicas, centros, institutos de investigación, redes y proyectos tan valiosos como el Siedna, que favorecen el trabajo interdisciplinario, como también el interuniversitario, con sedes y recintos como participantes directos y activos, los que a la vez, facilitan el intercambio con las comunidades. Pero también se han vigorizado las alianzas con organismos nacionales (estatales, de la sociedad civil) e internacionales.

El siguiente cuadro da cuenta de estos vínculos:

12 El acuerdo es del 05/07/09. El CNNA es creado por mandato del CNA "como espacio de deliberación, concertación y coordinación entre el Poder Ejecutivo, las instituciones descentralizadas del Estado y las organizaciones representativas de la comunidad relacionadas con la materia" (capítulo 11, artículo $170^{\circ}$-Creación). Valga decir que la UCR ha tenido siempre una representación ante el CNNA. 


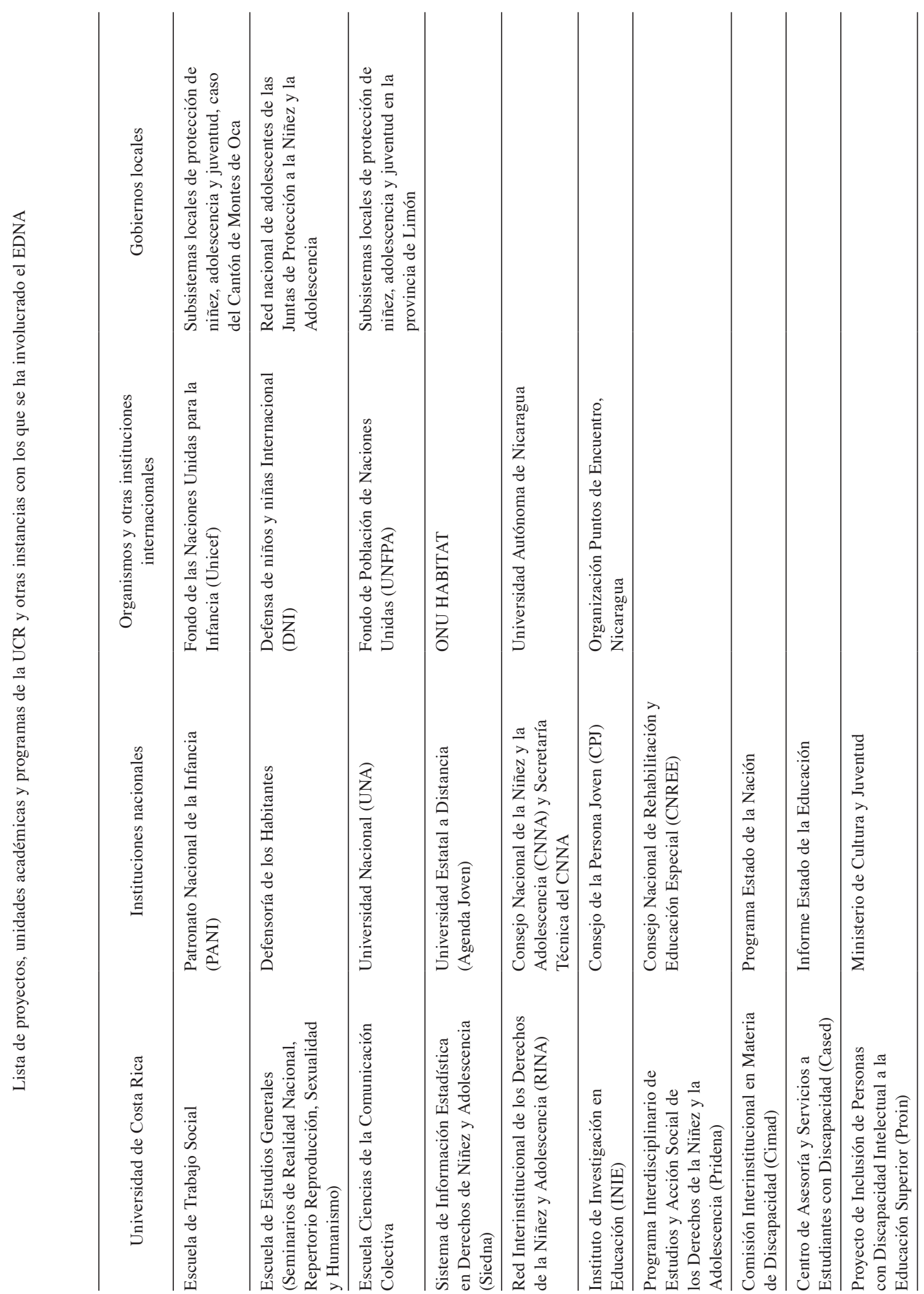




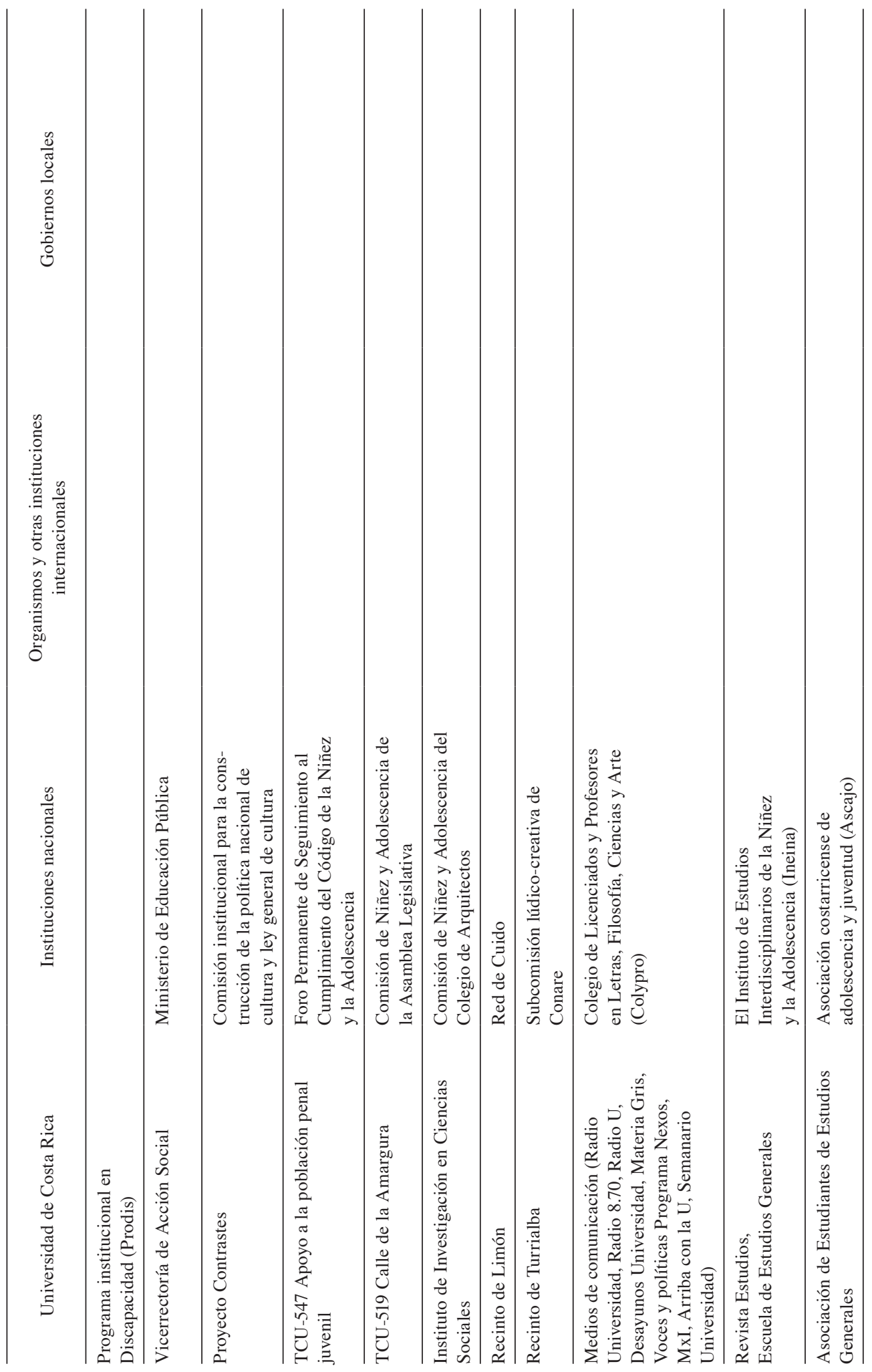


La continuidad del proyecto, y del Informe en particular, es un buen indicador del compromiso con el tema de los derechos humanos de las personas menores de edad, pero también, una buena coyuntura para revisar los alcances logrados y la estrategia por seguir, así como el nivel de participación real que tienen las personas menores de edad en procesos como este, que les afecta de manera directa.

Trabajar en derechos de las personas menores de edad supone abrir los espacios necesarios para que estas se incorporen de forma activa, propositiva y crítica según su edad, niveles de autonomía y capacidades físicas e intelectuales, como la experiencia ha demostrado que actúan, pero además, como la misma convención de los derechos del niño y la niña lo demandan. Esto tiene una serie de implicaciones que ha asumido el EDNA desde su concepción, y parte de lo aquí relatado se enraíza, precisamente, en el trabajo directo con niñas, niños y adolescentes para la gestación del Informe.

\section{Algunas ideas finales}

El Planes 2011-2015 Conare señala el compromiso social de las universidades y exhorta a la articulación de esfuerzos, de manera que se potencien las capacidades académicas e institucionales. Esta es una clara evidencia de que la academia no puede ser indiferente a su realidad nacional, regional y mundial. Es claro que invertir en niñez y adolescencia en el espacio académico es parte de nuestra responsabilidad social, porque hacerlo significa trabajar por una sociedad equitativa, inclusiva, ajena a la reproducción de círculos de pobreza, explotación e ignorancia.

El esfuerzo que la UCR ha hecho hasta la fecha para fortalecer diversas acciones vinculadas al tema de los derechos de la niñez y la adolescencia, en concreto la sostenibilidad para la continuidad del proyecto y del Informe EDNA, a lo largo de casi tres quinquenios, es una significativa evidencia de su compromiso como garante del bien común y del mejoramiento de la calidad de vida de las personas. Pero además, esta experiencia es un buen ejemplo del encuentro de las tres áreas centrales del quehacer universitario: docencia, acción social e investigación. ${ }^{13}$

\section{Referencias}

Castillo, Carmen; Arias, Gabriela y Zúñiga, Marcela. (2011). Aportes de la Universidad de Costa Rica en la promoción de los derechos humanos de la niñez y la adolescencia. Reflexiones, 90 (1), 121-134.

Código de la niñez y la adolescencia. (2010). San José, Costa Rica: Editorial Investigaciones Jurídicas, S.A.

Consejo Nacional de Rectores (CONARE). (2011). Plan Nacional de Educación Superior Universitaria Estatal 2011-2015.San José, Costa Rica. Recuperado dehttp://www.uned.ac.cr/images/rector/ PLAN_DE_ACCION_-_PLANES_mayo_2011.pdf

Coordinación EDNA. (2008).Informe anual de labores 2008 del proyecto ED-389 Estado de los derechos de la niñez y la adolescencia. San José: Universidad de Costa Rica. Manuscrito inédito.

Coordinación EDNA. (2009).Informe anual de labores 2009del proyecto ED-389 Estado de los derechos de la niñez y la adolescencia. San José: Universidad de Costa Rica. Manuscrito inédito.

Coordinación EDNA. (2010).Informe anual de labores 2010del proyecto ED-389 Estado de los derechos de la niñez y la adolescencia. San José: Universidad de Costa Rica. Manuscrito inédito.

Coordinación EDNA. (2011a).Informe anual de labores 2011del proyecto ED-389 Estado de los derechos de la niñez y la adolescencia. San José: Universidad de Costa Rica. Manuscrito inédito.

13 Se agradece el aporte brindado, para efectos de la elaboración de este documento, a las académicas María Pérez-Yglesias, Vicerrectora de Acción Social período 2004-2014; Carmen María Castillo, coordinadora técnica del Pridena; Sonia Solís, Coordinadora proyecto EDNA 2002, y a Ludwig Guendel, especialista en derechos de la niñez y la adolescencia, miembro del equipo gestor del proyecto EDNA. 
Coordinación EDNA. (2011b). Sistematización Semana joven 2011. San José: Universidad de Costa Rica. Manuscrito inédito.

Coordinación EDNA. (2012a).Informe anual de labores 2012del proyecto ED-389 Estado de los derechos de la niñez y la adolescencia. San José: Universidad de Costa Rica. Manuscrito inédito.

Coordinación EDNA. (2012b). Sistematización Semana joven 2012. San José: Universidad de Costa Rica. Manuscrito inédito.

Coordinación EDNA. (2013a).Informe anual de labores 2013del proyecto ED-389 Estado de los derechos de la niñez y la adolescencia. San José: Universidad de Costa Rica. Manuscrito inédito.

Coordinación EDNA.(2013b). Sistematización Semana joven 2013. San José: Universidad de Costa Rica. Manuscrito inédito.

Guendel, Ludwig; Barahona, Manuel y Bustelo, Emilio. (2005). Derechos humanos, niñez y adolescencia. Cuaderno de Ciencias Sociales N 138. San José, Costa Rica: Facultad Latinoamericana de Ciencias Sociales.

Organización de las Naciones Unidas (ONU). (1989). Convención sobre los derechos del niño. Recuperado dehttp://www.unicef.org/argentina/spanish/7.-Convencionsobrelosderechos.pdf

Patronato Nacional de la Infancia (PANI); Fondo de las Naciones Unidas para la Infancia (Unicef). (2009). Política nacional para la niñez y la adolescencia Costa Rica 2009-2021. San José, Costa Rica.

Programa Interdisciplinario de Estudios y Acción Social de los Derechos de la Niñez y la Adolescencia (PRIDENA). (2008). Plan estratégico PRIDENA 2008-2012. San José, Costa Rica. Manuscrito inédito.

Universidad de Costa Rica (UCR); Fondo de las Naciones Unidas para la Infancia (Unicef). (2001). I Estado de los derechos de la niñez y la adolescencia. Unicef; Universidad de Costa Rica. San José, Costa Rica.

Universidad de Costa Rica (UCR); Fondo de las Naciones Unidas para la Infancia (Unicef). (2002). II Estado de los derechos de la niñez y la adolescencia. Unicef; Universidad de Costa Rica. San José, Costa Rica.

Universidad de Costa Rica (UCR); Fondo de las Naciones Unidas para la Infancia (Unicef). (2003). III Estado de los derechos de la niñez y la adolescencia. Unicef; Universidad de Costa Rica. San José, Costa Rica.

Universidad de Costa Rica (UCR); Fondo de las Naciones Unidas para la Infancia (Unicef). (2004). IV Estado de los derechos de la niñez y la adolescencia. Unicef; Universidad de Costa Rica. San José, Costa Rica.

Universidad de Costa Rica (UCR); Fondo de las Naciones Unidas para la Infancia (Unicef). (2005). V Estado de los derechos de la niñez y la adolescencia. Unicef; Universidad de Costa Rica. San José, Costa Rica.

Universidad de Costa Rica (UCR); Fondo de las Naciones Unidas para la Infancia (Unicef). (2008). VI Estado de los derechos de la niñez y la adolescencia. Unicef; Universidad de Costa Rica. San José, Costa Rica.

Universidad de Costa Rica (UCR); Fondo de las Naciones Unidas para la Infancia (Unicef). (2011). VII Estado de los derechos de la niñez y la adolescencia. Unicef; Universidad de Costa Rica. San José, Costa Rica. 
